\title{
Molecular Parameters for the Band Systems A, B-X of AuH and A-X of AuD
}

\author{
N. Rajamanickam, M. Vignesh Kumar, V. Raja, and B. Karthikeyan \\ Physics Research Centre, VHNSN College, Virudhunagar - 626 001, India
}

Received on 18 October, 2006

\begin{abstract}
Franck-Condon factors (vibrational transition probabilities) and r-centroids have been evaluated by a numerical integration procedure for the bands of $\mathrm{A}^{+g}-\mathrm{X}^{1} \Sigma^{+}$, B $0^{+g}-\mathrm{X}^{1} \Sigma^{+}$systems of $\mathrm{AuH}$ and $\mathrm{A} 0^{+}-$ $\mathrm{X}^{1} \Sigma^{+}$system of $\mathrm{AuD}$ molecules using a suitable potential. The physical and astrophysical significances of our evaluated Franck-Condon factors and r-centroids have also been discussed, which would be helpful in the prediction of the presence of $\mathrm{AuH}$ and $\mathrm{AuD}$ molecules in various celestial bodies.
\end{abstract}

Keywords: Franck-Condon factors; r-centroids; AuH and AuD molecules

\section{INTRODUCTION}

The theoretical prediction of intensity distribution in the molecular band system requires a knowledge of molecular parameters like vibrational transition probabilities, which are to a good approximation proportional to the Franck-Condon (FC) factors and r-centroids. A precise knowledge of FC factors and related quantities are essential for understanding and calculating many important data for the molecules, e.g., radiative lifetime, vibrational temperature and kinetics of energy transfer. Both the molecular parameters that is FC factors and r-centroids play an important role to arrive at the variation of electronic transition moment with internuclear separation, relative band strength and vibrational temperature of the source [1-2]. The vibrational transition probability parameters for the diatomic molecules are therefore necessary for diagnostic applications in astronomy, astrophysics and allied subjects.

The gold atom and its compounds are well-known examples for the influence of relativistic effects on atomic and molecular properties [3-5]. In astrophysical application, Johnson et al. [6] calculated the weighted column densities for 248 molecules including AuH which could be possibly present in an oxygen-rich (O-rich) star under the effective temperature $2500 \leq T_{\text {eff }} \leq 4000 \mathrm{~K}$.

As there has been no report on the FC factors and $\mathrm{r}$ centroids for the emission band systems $\mathrm{A}^{+g}-\mathrm{X}^{1} \Sigma^{+}, \mathrm{B}$ $0^{+g}-\mathrm{X}^{1} \Sigma^{+}$of $\mathrm{AuH}$ and $\mathrm{A} 0^{+}-\mathrm{X}^{1} \Sigma^{+}$of $\mathrm{AuD}$, we have computed the values of FC fators and r-centroids for these band systems by the numerical integration procedure using a suitable potential.

\section{THEORY}

The intensity $\left(I_{v^{\prime}} v^{\prime \prime}\right)$ of a molecular band for an electronic transition in emission is

$$
I_{v^{\prime} v^{\prime \prime}}=D N_{v^{\prime}} E_{v^{\prime} v^{\prime \prime}}^{4} p_{v^{\prime} v^{\prime \prime}}
$$

where $D$ is a constant partly depending on the geometry of the apparatus, $N_{v^{\prime}}$ is the population of the level $v^{\prime}, E_{v^{\prime} v^{\prime \prime}}\left(=\lambda_{v^{\prime} v^{\prime \prime}}^{-1}\right)$ is the quantum energy and $p_{v^{\prime} v^{\prime \prime}}$ is the band strength. In terms of the electronic transition moment $R_{e}, p_{v^{\prime} v^{\prime \prime}}$ is given by

$$
p_{v^{\prime} v^{\prime \prime}}=R_{e}^{2}\left(\bar{r}_{v^{\prime} v^{\prime \prime}}\right) q_{v^{\prime} v^{\prime \prime}}
$$

The quantities $q_{v^{\prime} v^{\prime \prime}}$ and $\bar{r}_{v^{\prime} v^{\prime \prime}}$ are the Franck-Condon factors and r-centroids respectively. The form of $q_{v^{\prime} v^{\prime \prime}}$ can be expressed as the square of the vibrational overlap integral

$$
q_{v^{\prime} v^{\prime \prime}}=\left|\left\langle\psi_{v^{\prime}} \mid \psi_{v^{\prime \prime}}\right\rangle\right|^{2}
$$

where $\psi_{v^{\prime}}$ and $\psi_{v^{\prime \prime}}$ are the vibrational wave functions for the upper and lower states respectively. The r-centroid $\left(\bar{r}_{v^{\prime}} v^{\prime \prime}\right)$ represents the characteristic internuclear separation of $v^{\prime}-v^{\prime \prime}$ transition in a diatomic molecular band system and is defined by

$$
\bar{r}_{v^{\prime} v^{\prime \prime}}=\frac{\left\langle\psi_{v^{\prime}}|r| \psi_{v^{\prime \prime}}\right\rangle}{\left\langle\psi_{v^{\prime}} \mid \psi_{v^{\prime \prime}}\right\rangle}
$$

Keeping these in view, the FC factors and r-centroids for some electronic band systems of AuH and AuD molecules could be computed as per the procedure described below.

\section{COMPUTATIONAL PROCEDURE}

For a proper understanding of the intensity distribution in the band system of the molecule, it is necessary to choose a suitable potential. The potential energy curves for the electronic states of $\mathrm{A}, \mathrm{B}, \mathrm{X}$, of $\mathrm{AuH}$ and $\mathrm{A}, \mathrm{X}$ of $\mathrm{AuD}$ molecules have been constructed using Morse [7] function and also by Rydberg-Klein-Rees (RKR) procedure as modified by Vanderslice et al. [8]. The vibrational energy $G(v)$, the turning points $r_{\text {max,min }}$ data are reported in tables I to V. It is found that the Morse function represents the potential energy curves of all the electronic states quite adequately, since the RKR curve is nearly coincident with the Morse one.

The Morse functions are then calculated at the intervals of $0.01 \AA$ for ' $r$ ' ranging from $1.23 \AA$ to $1.43 \AA$, from $1.25 \AA$ to $2.54 \AA$ and from $1.24 \AA$ to $2.94 \AA$ for every observed vibrational level of each state of $\mathrm{A}-\mathrm{X}, \mathrm{B}-\mathrm{X}$ systems of $\mathrm{AuH}$ and $\mathrm{A}-\mathrm{X}$ system of $\mathrm{AuD}$ molecules. Once the appropriate wave functions are obtained, the FC factors can be determined by evaluating the integral in equation (3). The definition of r-centroid offers a method of computing r-centroids directly. The computation of the FC factors is made by Bates' [9] method of numerical integration according to the detailed procedure provided by Partal Urena et al [10].

The integrals in the equations (3) and (4) for the FC factors $\left(q_{v^{\prime} v^{\prime \prime}}\right)$ and r-centroids $\left(\bar{r}_{v^{\prime} v^{\prime \prime}}\right)$ are computed numerically for 
TABLE I: Turning points for the molecular vibration in the A-state of $\mathrm{AuH}$

\begin{tabular}{|c|c|c|c|c|c|}
\hline & & \multicolumn{2}{|c|}{ Morse } & \multicolumn{2}{c|}{ RKR } \\
\cline { 3 - 6 } $\mathrm{v}$ & $\mathrm{G}(\mathrm{v})$ in $\mathrm{cm}^{-1}$ & $r_{\max }$ in $\AA$ & $r_{\min }$ in $\AA$ & $r_{\max }$ in $\AA$ & $r_{\min }$ in $\AA$ \\
\hline 0 & 820.52 & 1.835 & 1.548 & 1.835 & 1.547 \\
1 & 2367.18 & 1.986 & 1.474 & 1.998 & 1.470 \\
2 & 3768.34 & 2.109 & 1.432 & 2.120 & 1.421 \\
3 & 5000.44 & 2.220 & 1.403 & 2.259 & 1.376 \\
4 & 6039.89 & 2.322 & 1.383 & 2.406 & 1.335 \\
5 & 6863.11 & 2.410 & 1.368 & 2.554 & 1.297 \\
\hline
\end{tabular}

TABLE II: Turning points for the molecular vibration in the B-state of AuH

\begin{tabular}{|c|c|c|c|c|c|}
\hline & & \multicolumn{2}{|c|}{ Morse } & \multicolumn{2}{c|}{ RKR } \\
\cline { 3 - 6 } $\mathrm{V}$ & $\mathrm{G}(\mathrm{v})$ in $\mathrm{cm}^{-1}$ & $r_{\max }$ in $\AA$ & $r_{\min }$ in $\AA$ & $r_{\max }$ in $\AA$ & $r_{\min }$ in $\AA$ \\
\hline 0 & 753.75 & 1.869 & 1.568 & 1.862 & 1.561 \\
1 & 2150.25 & 2.042 & 1.497 & 2.017 & 1.472 \\
2 & 3398.75 & 2.195 & 1.457 & 2.151 & 1.413 \\
3 & 4499.25 & 2.351 & 1.429 & 2.284 & 1.362 \\
4 & 5451.75 & 2.519 & 1.409 & 2.425 & 1.315 \\
\hline
\end{tabular}

TABLE III: Turning points for the molecular vibration in the X-state of $\mathrm{AuH}$

\begin{tabular}{|c|c|c|c|c|c|}
\hline & & \multicolumn{2}{|c|}{ Morse } & \multicolumn{2}{c|}{ RKR } \\
\cline { 3 - 6 } $\mathrm{v}$ & $\mathrm{G}(\mathrm{v})$ in $\mathrm{cm}^{-1}$ & $r_{\max }$ in $\AA$ & $r_{\min }$ in $\AA$ & $r_{\max }$ in $\AA$ & $r_{\min }$ in $\AA$ \\
\hline 0 & 1141.72 & 1.657 & 1.414 & 1.658 & 1.415 \\
1 & 3360.35 & 1.774 & 1.346 & 1.776 & 1.348 \\
2 & 5492.34 & 1.866 & 1.304 & 1.870 & 1.307 \\
3 & 7537.43 & 1.950 & 1.273 & 1.954 & 1.277 \\
\hline
\end{tabular}

TABLE IV: Turning points for the molecular vibration in the A-state of AuD

\begin{tabular}{|c|c|c|c|c|c|}
\hline & & \multicolumn{2}{|c|}{ Morse } & \multicolumn{2}{c|}{ RKR } \\
\cline { 3 - 6 } $\mathrm{v}$ & $\mathrm{G}(\mathrm{v})$ in $\mathrm{cm}^{-1}$ & $r_{\max }$ in $\AA$ & $r_{\min }$ in $\AA$ & $r_{\max }$ in $\AA$ & $r_{\min }$ in $\AA$ \\
\hline 0 & 588.92 & 1.804 & 1.563 & 1.803 & 1.562 \\
1 & 1714.53 & 1.928 & 1.500 & 1.924 & 1.496 \\
2 & 2770.52 & 2.030 & 1.463 & 2.023 & 1.455 \\
3 & 3756.88 & 2.127 & 1.436 & 2.115 & 1.424 \\
4 & 4673.62 & 2.223 & 1.415 & 2.206 & 1.398 \\
5 & 5520.73 & 2.321 & 1.398 & 2.298 & 1.375 \\
6 & 6298.21 & 2.423 & 1.384 & 2.393 & 1.354 \\
7 & 7006.07 & 2.532 & 1.372 & 2.494 & 1.334 \\
8 & 7644.30 & 2.650 & 1.362 & 2.601 & 1.314 \\
9 & 8212.91 & 2.779 & 1.354 & 2.718 & 1.294 \\
10 & 8711.89 & 2.923 & 1.347 & 2.848 & 1.272 \\
\hline
\end{tabular}

TABLE V: Turning points for the molecular vibration in the X-state of AuD

\begin{tabular}{|c|c|c|c|c|c|}
\hline & & \multicolumn{2}{|c|}{ Morse } & \multicolumn{2}{c|}{ RKR } \\
\cline { 3 - 6 } $\mathrm{v}(\mathrm{v})$ in $\mathrm{cm}^{-1}$ & $r_{\max }$ in $\AA$ & $r_{\min }$ in $\AA$ & $r_{\max }$ in $\AA$ & $r_{\min }$ in $\AA$ \\
\hline 0 & 811.82 & 1.635 & 1.430 & 1.635 & 1.430 \\
1 & 2401.40 & 1.729 & 1.370 & 1.730 & 1.371 \\
2 & 3945.41 & 1.802 & 1.333 & 1.803 & 1.335 \\
3 & 5443.67 & 1.867 & 1.305 & 1.869 & 1.307 \\
4 & 6896.02 & 1.927 & 1.283 & 1.930 & 1.285 \\
5 & 8302.28 & 1.986 & 1.264 & 1.988 & 1.266 \\
\hline
\end{tabular}


TABLE VI: $q_{v^{\prime} v^{\prime \prime}}$ and $\bar{r}_{v^{\prime} v^{\prime \prime}}$ of A-X band system of AuH

\begin{tabular}{|c|cc|c|c|c|c|}
\hline & & $v^{\prime \prime}=0$ & $v^{\prime \prime}=1$ & $v^{\prime \prime}=2$ & $v^{\prime \prime}=3$ & $v^{\prime \prime}=4$ \\
\hline$v^{\prime}=0$ & a) & 0.493 & 0.376 & 0.115 & 0.019 & 0.002 \\
& b) & 1.613 & 1.742 & 1.881 & 2.025 & 2.180 \\
& c) & 3656.99 & 3461.28 & 3301.29 & 3172.33 & - \\
\hline$v^{\prime}=1$ & a) & 0.302 & 0.030 & 0.338 & 0.250 & 0.073 \\
& b) & 1.522 & 1.679 & 1.783 & 1.917 & 2.059 \\
& c) & 3979.99 & 3749.19 & 3562.26 & 3412.58 & 3296.94 \\
\hline$v^{\prime}=2$ & a) & 0.129 & 0.188 & 0.039 & 0.142 & 0.308 \\
& b) & 1.443 & 1.573 & 1.665 & 1.831 & 1.958 \\
& c) & 4349.00 & 4047.90 & 3855.03 & - & - \\
\hline \multirow{2}{*}{$v^{\prime}=3$} & a) & 0.049 & 0.177 & 0.031 & 0.143 & 0.011 \\
& b) & 1.374 & 1.495 & 1.642 & 1.727 & 1.913 \\
& c) & 4773.54 & 3922.26 & 4183.21 & 3979.88 & - \\
\hline \multirow{2}{*}{$v^{\prime}=4$} & a) & 0.019 & 0.110 & 0.107 & 0.008 & 0.140 \\
& b) & 1.315 & 1.428 & 1.553 & 1.596 & 1.783 \\
& c) & - & 4869.00 & 4558.36 & - & - \\
\hline \multirow{2}{*}{$v^{\prime}=5$} & a) & 0.007 & 0.059 & 0.115 & 0.017 & 0.075 \\
& b) & 1.264 & 1.371 & 1.487 & 1.637 & 1.696 \\
& c) & - & - & - & 4964.86 & - \\
\hline
\end{tabular}

TABLE VII: $q_{v^{\prime} v^{\prime \prime}}$ and $\bar{r}_{v^{\prime} v^{\prime \prime}}$ of B-X band system of AuH

\begin{tabular}{|c|cc|c|c|c|}
\hline & & $v^{\prime \prime}=0$ & $v^{\prime \prime}=1$ & $v^{\prime \prime}=2$ & $v^{\prime \prime}=3$ \\
\hline \multirow{2}{*}{$v^{\prime}=0$} & a) & 0.386 & 0.395 & 0.172 & 0.045 \\
& b) & 1.624 & 1.740 & 1.864 & 1.986 \\
& c) & 2361.98 & 2281.73 & - & - \\
\hline$v^{\prime}=1$ & a) & 0.294 & $*$ & 0.211 & 0.292 \\
& b) & 1.550 & & 1.785 & 1.904 \\
& c) & 2492.61 & & 2428.83 & - \\
\hline$v^{\prime}=2$ & a) & 0.158 & 0.080 & 0.127 & 0.009 \\
& b) & 1.488 & 1.614 & 1.706 & 1.847 \\
& c) & 2715.83 & 2615.62 & 2567.17 & 2514.06 \\
\hline \multirow{2}{*}{$v^{\prime}=3$} & a) & 0.078 & 0.126 & 0.007 & 0.122 \\
& b) & 1.436 & 1.552 & 1.597 & 1.766 \\
& c) & 2822.84 & 2776.77 & - & - \\
\hline \multirow{2}{*}{$v^{\prime}=4$} & a) & 0.038 & 0.110 & 0.010 & 0.090 \\
& b) & 1.394 & 1.503 & 1.653 & 1.705 \\
& c) & 2875.51 & - & - & - \\
\hline
\end{tabular}

the bands of $\mathrm{A}-\mathrm{X}, \mathrm{B}-\mathrm{X}$ systems of $\mathrm{AuH}$ and $\mathrm{A}-\mathrm{X}$ system of $\mathrm{AuD}$ molecules and the results are tabulated respectively in tables VI to VIII. The available wavelengths data $\left(\lambda_{v^{\prime} v^{\prime \prime}}\right)$ [11-13] are also entered. The molecular constants used in the present study are collected from the compilation of Huber and Herzberg [14].

\section{RESULTS AND DISCUSSION}

The FC factors indicate that the $(0,0),(0,1),(1,0),(1,2)$, $(1,3)$ and $(2,4)$ bands are bright and all the other bands are comparatively weak for the band system of $\mathrm{A}-\mathrm{X}$ of $\mathrm{AuH}$. In the case of $\mathrm{B}-\mathrm{X}$ band system of $\mathrm{AuH}, \mathrm{FC}$ factors divulge that $(0,0),(0,1),(1,0),(1,2)$ and $(1,3)$ bands are strong when compared to other bands. On investigating the FC factors for the band systems $\mathrm{A}-\mathrm{X}$ of AuD molecule, it is found that the $(0,0)$,
$(0,1),(1,0),(1,2),(1,3),(2,4)$ and $(2,5)$ bands are prominent while all the other bands are weak. We have obtained the FC factor ratio (intensity ratio) for the $(0,0)$ band in $\mathrm{A}-\mathrm{X}$ system as $1: 0.7819$ of $\mathrm{AuH}: \mathrm{AuD}$.

The sequence differences for the $\mathrm{A}-\mathrm{X}$ and $\mathrm{B}-\mathrm{X}$ band systems of AuH are found to be varying from $0.014 \AA$ to 0.084 $\AA$ and from $0.040 \AA$ to $0.067 \AA$ respectively. In case of $\mathrm{A}-\mathrm{X}$ band system of $\mathrm{AuD}$, the sequence differences are found to be varying in between $0.001 \AA$ to $0.070 \AA$.

In all the systems discussed above, $\bar{r}_{00}$ is greater than $\left(r_{e}^{\prime}+\right.$ $\left.r_{e}^{\prime \prime}\right) / 2$ by $0.01 \AA$ which reveals that the potentials B, A \& X of $\mathrm{AuH}$ and $\mathrm{A} \& \mathrm{X}$ of $\mathrm{AuD}$ are more anharmonic. 
TABLE VIII: $q_{v^{\prime} v^{\prime \prime}}$ and $\bar{r}_{v^{\prime} v^{\prime \prime}}$ of A-X band system of AuD

\begin{tabular}{|c|c|c|c|c|c|c|c|}
\hline & & $v^{\prime \prime}=0$ & $v^{\prime \prime}=1$ & $v^{\prime \prime}=2$ & $v^{\prime \prime}=3$ & $v^{\prime \prime}=4$ & $v^{\prime \prime}=5$ \\
\hline \multirow{3}{*}{$v^{\prime}=0$} & a) & 0.385 & 0.398 & 0.171 & 0.042 & 0.007 & 0.001 \\
\hline & b) & 1.606 & 1.699 & 1.798 & 1.901 & 2.004 & 2.109 \\
\hline & c) & 3646.80 & 3503.03 & 3378.12 & 3269.90 & 3177.02 & - \\
\hline \multirow{3}{*}{$v^{\prime}=1$} & a) & 0.310 & * & 0.231 & 0.289 & 0.133 & 0.034 \\
\hline & b) & 1.541 & & 1.729 & 1.825 & 1.925 & 2.026 \\
\hline & c) & 3871.44 & & - & - & 3346.17 & 3258.96 \\
\hline \multirow{3}{*}{$v^{\prime}=2$} & a) & 0.166 & 0.107 & 0.112 & 0.030 & 0.245 & 0.225 \\
\hline & b) & 1.484 & 1.582 & 1.652 & 1.769 & 1.854 & 1.952 \\
\hline & c) & 4118.40 & 3935.97 & 3778.96 & - & - & - \\
\hline \multirow{3}{*}{$v^{\prime}=3$} & a) & 0.077 & 0.159 & * & 0.157 & 0.018 & 0.098 \\
\hline & b) & 1.434 & 1.522 & & 1.692 & 1.768 & 1.887 \\
\hline & c) & 4390.26 & 4183.56 & & 3855.29 & - & - \\
\hline \multirow{3}{*}{$v^{\prime}=4$} & a) & 0.035 & 0.129 & 0.046 & 0.062 & 0.061 & 0.108 \\
\hline & b) & 1.391 & 1.473 & 1.573 & 1.623 & 1.734 & 1.815 \\
\hline & c) & - & 4455.83 & 4255.66 & - & - & - \\
\hline \multirow{3}{*}{$v^{\prime}=5$} & a) & 0.016 & 0.084 & 0.087 & 0.001 & $0.106 \mathrm{~s}$ & * \\
\hline & b) & 1.355 & 1.430 & 1.518 & 1.442 & 1.671 & \\
\hline & c) & - & - & 4528.68 & 4336.29 & - & \\
\hline \multirow{3}{*}{$v^{\prime}=6$} & a) & 0.007 & 0.050 & 0.090 & 0.014 & 0.051 & 0.048 \\
\hline & b) & 1.324 & 1.394 & 1.474 & 1.587 & 1.612 & 1.721 \\
\hline & c) & - & - & - & - & - & 5451.49 \\
\hline \multirow{3}{*}{$v^{\prime}=7$} & a) & 0.004 & 0.029 & 0.074 & 0.040 & 0.008 & 0.075 \\
\hline & b) & 1.299 & 1.361 & 1.438 & 1.529 & 1.534 & 1.668 \\
\hline & c) & - & - & - & 4911.37 & 4704.80 & - \\
\hline \multirow{3}{*}{$v^{\prime}=8$} & a) & 0.002 & 0.017 & 0.054 & 0.052 & 0.001 & 0.051 \\
\hline & b) & 1.228 & 1.337 & 1.407 & 1.490 & 1.765 & 1.621 \\
\hline & c) & - & - & - & - & 5007.29 & - \\
\hline \multirow{3}{*}{$v^{\prime}=9$} & a) & 0.001 & 0.010 & 0.037 & 0.051 & 0.009 & 0.021 \\
\hline & b) & 1.260 & 1.316 & 1.381 & 1.458 & 1.569 & 1.573 \\
\hline & c) & - & - & - & - & 5338.20 & - \\
\hline \multirow{3}{*}{$v^{\prime}=10$} & a) & 0.001 & 0.006 & 0.025 & 0.044 & 0.019 & 0.005 \\
\hline & b) & 1.246 & 1.298 & 1.360 & 1.433 & 1.525 & 1.510 \\
\hline & c) & - & - & - & - & - & 5451.49 \\
\hline
\end{tabular}

a) $q_{v^{\prime} v^{\prime \prime}}$; b) $\bar{r}_{v^{\prime} v^{\prime \prime}}(\AA)$; c) Wavelength $\lambda_{v^{\prime} v^{\prime \prime}}(\AA) ;{ }^{*} q_{v^{\prime} v^{\prime \prime}} \approx 0$

\section{CONCLUSION}

The reported Franck-Condon factor and r-centroid values could be considered as satisfactory and reliable as they have been evaluated by the numerical integration procedure with the suitable potential function. The FC factors are directly proportional to the intensities of the bands and the intensity ratios reflect the abundance of the molecules [15]. Hence the above indicated intensity ratio may be interpreted as if $\mathrm{AuH}$ is present in an astrophysical object, there is about 78 percent possibility for the existence of AuD molecule also. For example, as already mentioned, AuH is predicted to exist in the O-rich star [6]. If the presence of AuH is confirmed in O-rich star, one may expect the presence of AuD molecule too in the same source. Therefore, the present study results are significant.
[1] N. Rajamanickam, J. Quant. Spectrosc. Radiat. Transfer 37, 207 (1987).

[2] N. Rajamanickam, Acta Phys. Hung. 63, 341 (1988).

[3] J. P. Desclanx and P. Pyykko, Chem. Phys. Lett. 39, 300 (1976).

[4] Y. Watanabe and O. Matsuoka, J. Chem. Phys. 116, 9585 (2002).

[5] I. Nakajima, T. Hanal, and K. Hirao, J. Comput. Chem. 23, 847 (2002).
[6] H.R. Johnson and A.J. Sauval, Astron. Astrophys. Suppl. Ser. 49, 77 (1982).

[7] P. M. Morse, Phys. Rev. 34, 57 (1929).

[8] J. T. Vanderslice, E. A. Mason, W. G. Maisch, and E. R. Lippincott, J. Mol. Spectr. 5, 83 (1960).

[9] D. R. Bates, Proc. Rov. Soc. A196, 217 (1949).

[10] F. Partal Urena, M. Fernandez Gomez, J.J. Lopez Gonzalez, and N. Rajamanickam, Astrophys. Space Sci. 272, 345 (2000). 
[11] T. Heimer, Zeitschrift fur Physik 101, 121 (1936).

[12] T. Heimer, Zeitschrift fur Physik 104, 303 (1937).

[13] U. Ringstrom, Arkiv foer Fysik 27, 227 (1964).

[14] K. P. Huber and G. Herzberg, Molecular Spectra and Molecular Structure, Vol. IV Constants of Diatomic Molecules, Van
Nostrand Reinhold, NewYork (1979).

[15] B. P. Straughan and S. Walker, Spectroscopy, Vol. 3, Chapmanc \& Hall, London (1976). 